\title{
Competition and cooperation between nodes in Delay Tolerant Networks with Two Hop Routing
}

\author{
Eitan Altman
}

INRIA B.P.93, 2004 Route des Lucioles, 06902 Sophia-Antipolis Cedex, France

\begin{abstract}
This paper revisits the two-hop forwarding policy in delay tolerant networks (DTNs) and provides a rich study of their performance and optimization which includes (i) Derivation of closed form expressions for the main performance measures such as success delivery probability of a packet (or a message) within a given deadline. (ii) A study of competitive and cooperative operations of DTNs and derivation of the structure of optimal and of equilibrium policies. (iii) A study of the case in which the entity that is forwarded is a chunk rather than a whole message. For a message to be received successfully, all chunks of which it is composed have to arrive at the destination within the deadline. (iv) A study of the benefits of adding redundant chunks. (v) The convergence to the mean field limit.
\end{abstract}

\section{Introduction}

Through mobility of devices that serve as relays, Delay Tolerant Networks (DTNs) allow non connected nodes to communicate with each other. Such networks have been developed in recent years and adapted both to human mobility where the contact process is between pedestrians [5], as well as to vehicle mobility [7].

The source does not know which of the nodes that it meets will reach the destination within a requested time, so it has to send many copies in order to maximize the successful delivery probability. How should it use its limited energy resources for efficient transmission? Assume that the first relay node to transfer the copy of the packet to the destination will receive a reward, or that some reward is divided among the nodes that participated in forwarding the packet. With what probability should a mobile participate in the forwarding, what is the optimal population size of mobiles when taking into account energy and/or other costs that increase as the number of nodes increase? If it is costly to be activated, how should one control the activation periods?

We propose in this paper some answers to these questions using simple probabilistic arguments. We identify structural properties of both static and dynamic optimal policies, covering both cooperative and non cooperative scenarios.

This paper pursues the research initiated in [2] where the authors already studied the optimal static and dynamic control problems using a fluid model that represents the mean field limit as the number of mobiles becomes very large. That work has been extended in [3] to model the separable nature of 
a packet, which is composed of $K$ blocks called chunks. Only once all chunks corresponding to a packet are received, the packet is considered to be available at the destination. The authors of [3] also study adding $H$ redundant chunks such that the packet can be reconstructed at the destination once it receives any $K$ out of the $K+H$ chunks.

In this paper we revisit the work of $[2,3]$ with the following differences. (i) In [3] it is assumed that memory is limited so that a mobile can only carry one chunk. In this paper we restrict to systems that do not have such constraints. (ii) Both problems $[2,3]$ were modeled using a mean field limit. We consider here the exact models and show that the mean field limit serves as a bound for the performance of the original system. The bound becomes tight as the number of mobiles increases.

In [4], a related optimal dynamic control problem was solved in a discrete time setting. The optimality of a threshold type policy, already established in [2] for the fluid limit framework, was shown to hold in [4] for some discrete control problem. A game problem between two groups of DTN networks was further studied in [4]. We complement these in the current work by focusing on other types of game theoretical problems: those concerning competition between individual mobiles. We obtain the structure of equilibrium policies and compare them to the cooperative case.

\section{$2 \quad$ Model}

Consider $n$ mobiles, and moreover, a single static source and destination. The source has a packet generated at time 0 that it wishes to send to the destination. Assume that any two mobiles meet each other according to a Poisson process with parameter $\lambda$. Whenever the mobile meets the source, the source may forward a packet to it. We consider the two hop routing scheme [1] in which a mobile that receives a copy of the packet from the source can only forward it if it meets the destination. It cannot copy it into the memory of another mobile.

Consider an active mobile with non-controlled transmission rate. Let $T_{1}$ be the first time it meets the source and let $T_{1}+T_{2}$ be the first time after $T_{1}$ that it meets the destination. Denote

$$
q_{\rho}=\exp (-\lambda \rho), \quad Q_{\rho}=Q_{\rho}(\lambda)=(1+\lambda \rho) \exp (-\lambda \rho)
$$

Consider the event that the mobile relays a packet from the source to the destination within time $\rho$, i.e. $T_{1}+T_{2} \leq \rho . T_{1}+T_{2}$ is an $\operatorname{Erlang}(2)$ random variable and therefore the probability of the above event is $1-Q_{\rho}$. Note that $Q_{\rho}-q_{\rho}$ is the probability that $T_{1}<\rho$ but that $T_{1}+T_{2}>\rho$.

\section{Control problems.}

We consider central control, in which the source decides whether to transmit a packet when it is in contact with a mobile, and distributed control, in which the mobiles are those who take decisions, concerning both the transmission of packets to the destination as well as of receiving packets from the source. 
As energy is limited or costly, we either limit the number of active mobiles, or we control dynamically the transmission rate. The first case corresponds to a static optimization problem; the decision variable $u^{i}$ then stands for the probability of participation. The dynamic control problem in which one controls dynamically the transmission rates. An individual dynamic control $u^{i}$ of mobile $i$ then stands for a piecewise continuous function $u_{t}^{i}$ taking values in $[0,1]$.

We shall consider two control frameworks: a centralized control at the source, and a decentralized control at the mobiles. When dynamic control is applied at the source it will affect transmissions from the source to the mobiles. When mobiles control the transmission then both transmission from the source to the mobiles as well as those from the mobile to the destination are controlled ${ }^{1}$

Interpretation of a dynamic control policy. If a mobile meets the source at time $t$ then it receives a packet with probability $u_{t}^{i}$. Moreover, in the case of decentralized control at the mobiles, if at time $t$, a mobile has a packet and it meets the destination then it delivers the packet with probability $u_{t}^{i}$.

Define $\zeta_{t}(j)$ to be the indicator that the $j$ th mobile among the $n$ receives the packet during $[0, t] .\left\{\zeta_{t}(j)\right\}_{j}$ are i.i.d. with the expectation and the Laplace Stieltjes Transform (LST) given by:

$$
\begin{gathered}
w_{t}^{j}:=E\left[\zeta_{t}(j)\right]=1-\exp \left(-\lambda \int_{0}^{t} u_{s}^{j} d s\right) \\
E\left[\exp \left(-s \zeta_{t}(j)\right)\right]=w_{t}^{j} \exp (-s)+\left(1-w_{t}^{j}\right)=1-(1-\exp (-s)) w_{t}^{j} \\
=1-(1-\exp (-s))\left(1-\exp \left(-\int_{0}^{t} u_{s}^{j} d s\right)=g\left(\int_{0}^{t} u_{s}^{j} d s\right)\right.
\end{gathered}
$$

where $g(Z)=\exp (-s)+(1-\exp (-s)) \exp (-s Z)$.

The probability that the destination does not receive a packet from mobile $j$ by time $\tau$ is given by $E\left[\exp \left(-\lambda \int_{0}^{\tau} \zeta_{t}(j) d t\right)\right]$ in the case of control at the source, and by $E\left[\exp \left(-\lambda \int_{0}^{\tau} \zeta_{t}(j) u_{t}^{j} d t\right)\right]$ for the case of decentralized control by the mobiles.

Dynamic control at the source: A state representation. Let $X_{t}$ be the number of mobiles with a copy of the packet at time $t$. We call $X_{t}$ the state. The intensity of the process that counts the number of contacts between nodes with copies of the packet and the destination at time $t$ is $\lambda X_{t}$. Thus the number of contacts during the interval $[0, \tau]$ between the destination and mobiles that have copies of the packets is a Poisson random variable with intensity $\lambda \int_{0}^{\tau} X_{s} d s$. Consider the dynamic control policy $u$ assumed to be common to all mobiles. Then $X_{t}=\sum_{j=1}^{n} \zeta_{t}(j)$ where $\left\{\zeta_{t}(j)\right\}_{j}$ are i.i.d. The LST of $X_{t}$ satisfies

$$
X_{t}^{*}(\lambda):=E\left[e^{-\lambda X_{t}}\right]=E\left[e^{-\lambda\left(\sum_{i=1}^{n} \zeta_{t}(i)\right)}\right]=\left(E\left[e^{-\lambda \zeta_{t}(1)}\right]\right)^{n}
$$

\footnotetext{
${ }^{1}$ also receiving packets require energy. This is in particular the case if a mobile that does not have the packet has to send periodically a beacon to signal its presence so that the source would know when it is in transmission range.
} 
Conditioned on $X_{t}$ for $t \in[0, \tau]$, the number of packets that arrive at the destination has a Poisson distribution with parameter $\lambda \int_{0}^{\tau} X_{t} d t$. Define $F_{D}(\tau)$ as the probability that the destination receives the packet by time $\tau$. Then

$$
F_{D}(\tau)=1-E\left[\exp \left(-\lambda \int_{0}^{\tau} X_{t} d t\right)\right]
$$

\section{The static DTN game}

It is assumed that the packet has to arrive at the destination $\tau$ units of time after it was created, otherwise it brings no utility to the destination. Let $u^{i}$ be the probability that mobile $i$ participates. $\left\{u^{i}, i=1, \ldots, n\right\}$ is a symmetric equilibrium if $u^{i}$ have the same value for all $i$ and if no mobile can benefit from a unilateral deviation to some $v \neq u$.

Let $p(v, u)$ be the probability that the tagged mobile is the first to deliver the packet to the destination when it plays $v$ and all others play $u$. A mobile other than the tagged one delivers a packet to the destination during the interval $[0, \tau]$ with probability $\beta:=u\left(1-Q_{\tau}\right)$. The probability that exactly $k-1$ mobiles other than the tagged one deliver a packet to the destination within the time interval is therefore: $\sum_{k=1}^{n}\left(\begin{array}{l}n-1 \\ k-1\end{array}\right) \beta^{k-1}(1-\beta)^{n-k}$. Hence the probability of the tagged mobile to receive the unit award, if it decides to participate, is

$$
\begin{aligned}
P(u) & =\left(1-Q_{\tau}\right) \sum_{k=1}^{n}\left(\begin{array}{c}
n-1 \\
k-1
\end{array}\right) \frac{\beta^{k-1}(1-\beta)^{n-k}}{k} \\
& =\frac{\left(1-Q_{\tau}\right)\left(1-(1-\beta)^{n}\right)}{\beta n}=\frac{\left(1-\left(1-\left(1-Q_{\tau}\right) u\right)^{n}\right)}{u n} .
\end{aligned}
$$

We shall sometime write $P_{n, \tau}(u)$ in order to make explicit the dependence on $n$ and $\tau$.

The utility and equilibrium A mobile that participates receives a unit of reward if it is the first to deliver a copy of the packet to the destination. It further pays some energy cost $g \tau$ where $g>0$ is some constant. $\mathcal{W}(1, u)=P(u)-g \tau$ is thus the (expected) utility for a tagged mobile of participating when each of the other mobiles participates with probability $u$. We assume that the utility $\mathcal{W}(0, u)$ for not participating is zero for all $u$. The utility for a mobile that participates with probability $v$ when each other participates with probability $u$ is $\mathcal{W}(v, u)=v \mathcal{W}(1, u)$. The following indifference property easily follows:

Lemma 1. If there exists a policy $u$ such that $\mathcal{W}(1, u)=0$ then $u$ is a symmetric equilibrium.

$P(u)$ is a continuous convex decreasing function, $\lim _{u \rightarrow 0} P(u)=1-Q_{\tau}$ and $P(1)=\left(1-Q_{\tau}^{n}\right) / n$. Thus the utility for choosing to participate $\mathcal{W}(1, u)=P(u)-$ $g \tau$ is a continuous convex decreasing function, $\lim _{u \rightarrow 0} \mathcal{W}(1, u)=1-Q_{\tau}-g(\tau)$ and $\mathcal{W}(1,1)=\left(1-Q_{\tau}^{n}\right) / n-g \tau$. The utility for not participating is assumed to be zero. Thus we have 
Lemma 2. If $\left(1-Q_{\tau}^{n}\right) / n<g \tau$ then there exists a unique symmetric equilibrium $u$ which is the unique solution of $\mathcal{W}(1, u)=\mathcal{W}(0, u)=0$.

Numerical Examples Figure 1 presents the utility for choosing to participate as a function of the strategy $u$ of all other players for various values of the maximum duration $\tau: \tau=1,5,20,60 . \lambda=10$ is taken to be a constant. The other parameters are $n=100, g=0.01$. We obtain four curves (one for each $\tau$ ). We see that indeed for each value of $\tau$ there is a unique value of $u$ for which $\mathcal{W}(1, u)=0$, and this is the equilibrium. Here the curve that is the highest corresponds to $\tau=1$, and the larger $\tau$ is, the lower the corresponding curve is. This then implies that the equilibrium value of $u$ increases with $\tau$.

In Figure 2 we repeat the same but with a fixed value $\tau=10$ and varying values of $n: n=3,10,30$ and 100 . Again, the larger $n$ is, the larger is also the curve. The equilibrium is thus increasing in $n$.

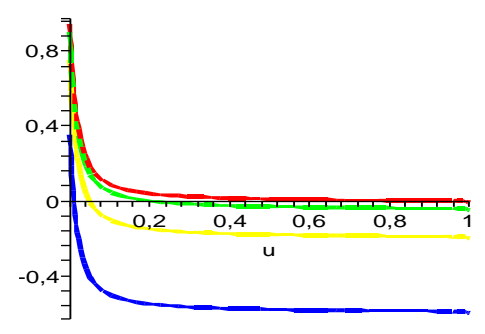

Fig. 1. The utility of participating as a function of $u$, for various values of the duration $\tau$.

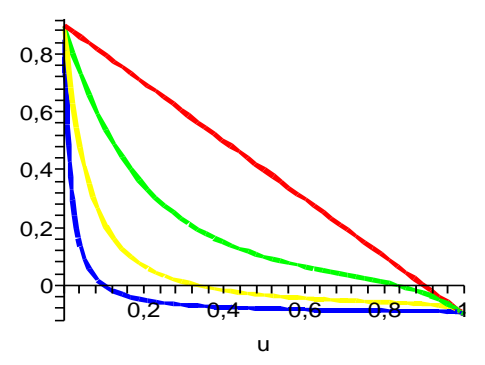

Fig. 2. The utility of participating as a function of $u$, for various values of the number $n$ of users.

\section{The static control problem}

We shall consider both the optimal symmetric control policy as well as the general non-symmetric one. We recall that we consider this as a problem in which the source controls the activation. A general activation policy is one that activates mobile $i$ with probability $u^{i}$. A symmetric policy is one for which $u^{i}$ are the same for all $i$. We consider here the utility

$$
\begin{gathered}
\mathcal{W}(u)=P_{s}(u)-g \tau \sum_{k=1}^{n} u^{k} \\
\text { where } \quad P_{s}(u)=1-\prod_{k=1}^{n}\left(u^{k} Q_{\tau}+\left(1-u^{k}\right)\right)=1-\prod_{k=1}^{n}\left(1-\left(1-Q_{\tau}\right) u^{k}\right)
\end{gathered}
$$


is the probability of successful transmission by time $\tau$. The term $g \tau \sum_{k=1}^{n} u^{k}$ corresponds to a cost per expected energy.

Let $U_{d}$ be the set of policies for which each $u^{k}$ is either 0 or 1 . We next show that the global utility for a general policy is minimized by a symmetric policy and maximized by a policy in $U_{d}$.

Lemma 3. (i) There exists an optimal policy among $U_{d}$.

(ii) A necessary condition for a policy $u$ to be globally optimal is the following:

Except for one mobile at most, the probability

of activating each mobile $i$ is either $u^{i}=0$ or $u^{i}=1$.

(iii) Any non-symmetric policy u performs strictly better than the symmetric policy $v$ that has the same sum $\sum_{k=1}^{n} u^{k}=\sum_{k=1}^{n} v^{k}$.

Proof. (i) Let $u$ be a policy for which for some $k, 0<u^{k}<1$. We show that there exists a policy $v \in U_{d}$ that performs at least as well. Since the utility is linear in each $u^{k}$, we can change $u^{k}$ to either 0 or to 1 without decreasing the utility. Repeating this procedure for all the remaining $j$ 's that are not extreme points, we obtain a policy in $U_{d}$ that performs at least as well as $u$. This implies (i).

Choose an arbitrary $u=\left(u^{1}, \ldots, u^{n}\right)$. Consider now the problem of finding $v \in$ $\mathcal{W}(u)$ under the constraint $\sum_{i=1}^{n} v^{k}=\sum_{i=1}^{n} u^{k}$. With this constraint, the policy maximizes this objective if and only if it minimizes the function $f(u)$ defined as

$$
f(u):=\sum_{k=1}^{n} \psi\left(u^{k}\right) \quad \text { where } \psi(u)=\log \left(1-\left(1-Q_{\tau}\right) u\right) .
$$

$\psi$ is a concave function of its argument, which implies that $f$ is a Schur concave function, see Appendix. For any policy $u$ which does not satisfy (3), we can construct a policy $u^{\prime}$ which satisfies (3) and $\sum_{k=1}^{n} u^{\prime k}=\sum_{k=1}^{n} u^{k}$. Then $u^{\prime}$ strictly majorizes $u$ and therefore $u$ strictly outperforms $u^{\prime}$. In the same way one shows that any policy performs strictly better than the symmetric policy that has the same sum of components.
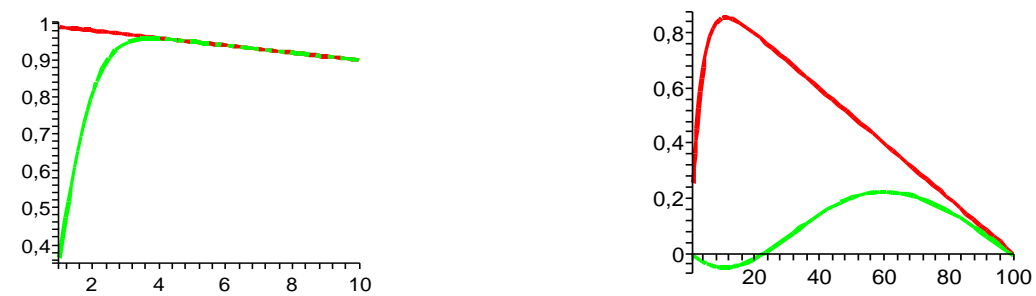

Fig. 3. Control case: Utility as a function of $k$ 
In Figure 3 we compare the best solution among the symmetric policies and the global optimal solution.

The left subfigure is obtained with the same parameters as used for the equilibrium in Figure 1: $g=1, n=100, \lambda=10, \tau=1$. The vertical axis corresponds to the utility, and the horizontal axis corresponds to the integer $k$. There are two curves. The top one is the utility under the non-symmetric policies, and the bottom is the one corresponding to symmetric ones. In the top figure, the integer $k$ has the meaning that exactly $k$ mobiles are activated. In the bottom curve, each mobile participates with probability $u$. $u$ is defined by the ratio $u=k / n$. $k$ varies between 1 to 10 .

The right subfigure repeats the same experiment but with $\lambda=1$ and with $n=100$. In both subfigures we see that there is indeed a difference between the global optimal solution and the one obtained with the best symmetric policy. The latter is indeed seen to provide a smaller optimum.

Remark 1. The fact that an optimal policy exists among $U_{d}$ means that there is an optimal number of mobiles that should participate. It can also be viewed as an optimal coalition size. We plan to study in the future the question of optimal coalition size in the case that there is competition between a given number $N$ of coalitions.

\section{The dynamic DTN game}

In the last section we assumed a static game: a mobile took one decision, at time 0 , on whether to participate or not. We now consider a dynamic game in which a mobile can switch on or off at any time. A pure policy $u^{i}$ for a mobile $i$ consists of the choice of time periods during which it is activated. $u_{t}^{i}=1$ if the mobile is activated at time $t$ and is zero if it is not. We may allow $u_{t}^{i}$ to take also values in the interior of the unit interval. $u_{t}^{i}$ is then interpreted as a mixed strategy: the mobile is activated at time $t$ with probability $u_{t}^{i}$. The energy cost for the mobile is given by $g \int_{0}^{\tau} u_{t}^{i} d t$.

We introduce next threshold policies. A time threshold policy $R$ is a policy that keeps a mobile active till time $R$ and then deactivates it. We shall identify threshold equilibria for our problem. More precisely, assume that all mobiles use threshold policies with a common threshold $R$. We shall consider deviation of a single mobile to another threshold policy $s$ and look for $R$ such that $s=R$ is an optimal response of the deviating mobile.

The probability that the deviating mobile is the first to deliver the packet to the destination is

$$
P_{\text {succ }}(s, R)=\left\{\begin{array}{lr}
\frac{1-\left(Q_{s}\right)^{n}}{n} & \text { if } s \leq R \\
\frac{1-\left(Q_{R}\right)^{n}}{n}+Q_{R}^{n-1}\left(q_{R}\left(1-Q_{s-R}\right)\right. & \\
\left.+\lambda R q_{R}\left(1-q_{s-R}\right)\right) & \text { otherwise }
\end{array}\right.
$$


The first term corresponds to the event that the first successful delivery occurs before time $R$, and the second term is related to its occurrence between time $R$ and $s$. More precisely, the second term corresponds to the event that no one of the other $n-1$ mobiles met the source till time $R$, where as the tagged mobile either (i) did not meet the source before time $R$ and then, during time interval $(R, s]$ met the source and then the destination, or (ii) it met the source at least once before $R$ but did not meet the destination before $R$, and then it met the destination during $(R, s]$.

The utility for a player who uses a threshold $s$ while all others use a threshold $R$ is given by $\mathcal{W}(s, R)=P_{\text {succ }}(s, R)-g s$.

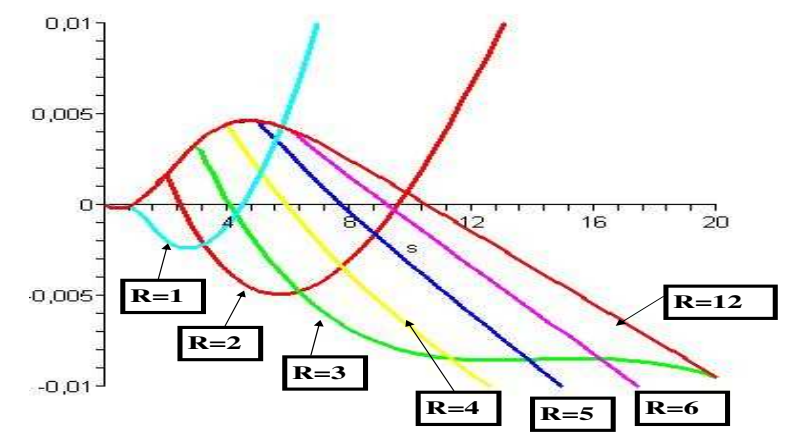

Fig. 4. Equilibria in threshold policies: The utility $\mathcal{W}(s, R)$ (vertical axis) for a mobile that uses a threshold policy $s$ (horizontal axis) while all the other use a threshold policy with $R$. Each curve corresponds to another value of $R$.

Figure 4 shows the utility $\mathcal{W}(s, R)$ for $s$ varying between 0 and 20 and for $R=1,2,3,4,5,6,12$. The horizontal axis corresponds to $s$ and the vertical axis - to the utility. The remaining parameters are $g=.001, \lambda=.049, n=95$. We observe the following.

- There are many equilibria. For $R$ between 3 and 5 , the best response is $s=R$ and hence any value of $R$ in the interval $[3,5]$ is a symmetric threshold equilibrium (for any horizon $\tau$ that is greater than $R$ ).

- $1 \leq R<3$ are also equilibria but only for some value of $\tau$. For example, for $R=2, s=2$ is the best response as long as we restrict $s$ to be smaller than 10.5; thus if $\tau \leq 10.5$ then all $R$ 's between 2 and 5 are symmetric threshold equilibria. If we restrict to $\tau \leq 4$ then the values of $R$ in the whole range $[1,5]$ provide symmetric equilibria.

An alternative way to see the multiple equilibria phenomenon is by plotting $\mathcal{W}(s, R)$ as a function of $R$ for various values of $s$. We do so in Figure 5 . This time we take $s=1,2,3,4,5,6,12$. The intersection of the curves corresponding to the different values of $s$ with the vertical axis are increasing with $s$. We indeed 
see that the best response to $R=3$ is $s=3$ but at the same time, $s=4$ is the best response to $R=4$. (To get the best response for $R=3$, we take a vertical line that intersects the horizontal axis at $R=3$. We see that the curve that corresponds to $s=3$ achieves the largest utility.)

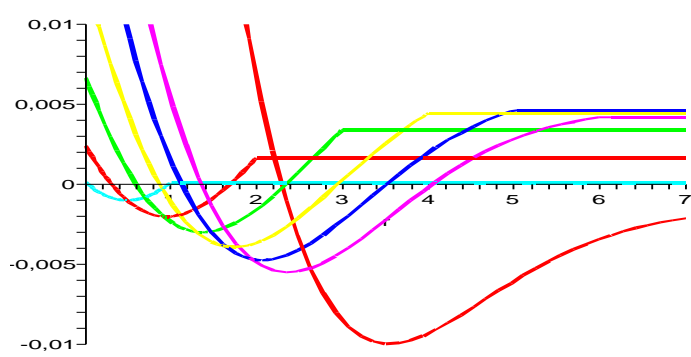

Fig. 5. Equilibria in threshold policies: The utility $\mathcal{W}(s, R)$ as a function of $R$ for various values of $s$.

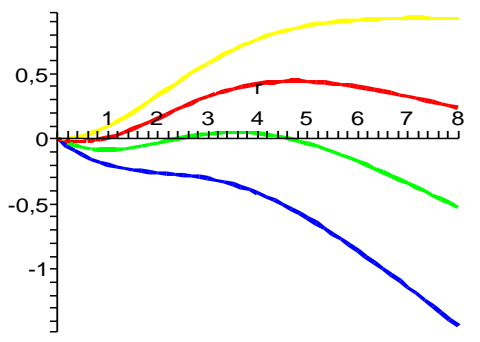

Fig. 6. The dynamic control case: utility as a function of $R$.

\section{The dynamic control problem}

We consider the optimization problem restricted to symmetric policies, i.e. where all mobiles use threshold policies and the threshold value $R$ is the same for all mobiles. Using the theory of Markov Decision Processes it can be shown that there is no loss of optimality in doing so.

The global utility is then $\mathcal{W}(R)=1-\left(Q_{R}\right)^{n}-n g R$. We have

$$
\frac{\partial \mathcal{W}(R)}{\partial R}=\frac{\left(Q_{R}\right)^{n} n \lambda^{2} R}{1+\lambda R}-n g, \quad \frac{\partial^{2} \mathcal{W}(R)}{\partial R^{2}}=\frac{n\left(Q_{R}\right)^{n} \lambda^{2}\left(n \lambda^{2} R^{2}-1\right)}{(1+\lambda R)^{2}}
$$

From the first derivative we see that $\mathcal{W}(R)$ is monotone. For all $R$ sufficiently large, it is negative if $g<1$ and is positive if $g>1$. From the second derivative we see that $\mathcal{W}(R)$ is convex for $R<1 /(\lambda \sqrt{n})$ and is concave for $R>1 /(\lambda \sqrt{n})$.

An example is given in Figure 6. The experiment was done with $n=95$. Each curve corresponds to another value of the parameter $g: g=0.0001$ (top curve), $g=0.0033$ (next to top), $g=0.001$ and $g=0.002$. We see that there may be one or two optimal values to the threshold $R$ : it is either an extreme point $(R=0$ or $R=\infty)$ or it is an interior point.

Theorem 1. There exists a unique optimal threshold policy. A policy is optimal only if (except for a set of Lebesgue measure zero) it agrees with this policy at all $t$. 
Proof With $X^{*}$ defined in (1), we have

$$
1-F_{D}(n)=E\left[\exp \left(-\lambda \int_{0}^{\tau} X_{t} d t\right)\right]=\exp \int_{0}^{\tau} \log \left(X_{t}^{*}(\lambda)\right) d t
$$

where the last equality follows from the Lévy Khinchine formula. Hence

$$
\begin{gathered}
P(\text { no success })=\exp \left(\int_{0}^{\tau} \log E\left[\exp \left(-\lambda X_{t}\right)\right] d t\right) \\
=\exp \left(n \int_{0}^{\tau} \log \gamma\left(\int_{0}^{t} u_{s} d s\right) d t\right) \\
\text { where } \gamma(Z):=e^{-\lambda}+\left(1-e^{-\lambda}\right) \exp (-\lambda Z)
\end{gathered}
$$

Assume that $u$ is not a threshold policy. Let $v$ be the threshold policy that transmits till time $s^{*}:=\int_{0}^{\tau} u_{t} d t$ and then stops transmitting. Then for every $t$,

$$
\int_{0}^{t} u_{s} d s \leq \int_{0}^{t} v_{s} d s
$$

This implies that the first integral $\int_{0}^{\tau}$ is smaller under $u$ and hence also the success probability.

A similar characterization of the optimal policy has been derived in [4] for the case of discrete time.

\section{Transmitting chunks}

We assume below that a message is composed of $K$ chunks. Consider some mobile that wishes to get the message within some time $\tau$. We wish to compute the success probability (given by the probability to receive all $K$ chunks within time $\tau$ ). Assume that there are initially $z_{i}$ nodes that have a copy of chunk $i$. It is assumed that the source can send at most one chunk when meeting another node. The dissemination of each chunk $i$ follows the same dynamics as the one described in Section 2. Thus the probability of successful delivery is given by

$$
\begin{gathered}
F_{D}=\prod_{j=1}^{K}\left(1-E\left[\exp \left(-\lambda \int_{0}^{\tau} X_{t}^{j} d t\right)\right]\right) \quad \text { so that } \\
\log F_{D}=\sum_{j=1}^{K} \log \mathbf{z}\left(u^{j}\right) \quad \text { where } \mathbf{z}\left(u^{j}\right)=1-\exp \left(n \int_{0}^{\tau} \log \gamma\left(\int_{0}^{t} u_{s}^{j} d s\right) d t\right)
\end{gathered}
$$

and where $\gamma(Z)$ is given in (4). The above derivation follows the same steps as in Theorem 1.

We shall study below "constant" transmission policies which are special case of dynamic policies, as well as general dynamic policies. With a constant transmission policy $u^{i}$, mobile $i$ is active. It receives a chunk from the source after an exponentially distributed time with parameter $u^{i} \lambda$. Once it has a chunk, it forwards it to the destination after an exponentially distributed time with parameter $\lambda$. 


\subsection{Optimal transmission policies}

Consider now the following problems:

- (P1) Find a constant policy $u^{i}$ that maximize $F_{D}-g \tau \sum_{i=1}^{K} u^{i}$,

- (P2) Find a dynamic policy $\mathbf{u}$ that maximizes $F_{D}-g \int_{0}^{\tau} \sum_{i=1}^{K} u_{s}^{i} d s$.

Lemma 4. (i) Let $u^{i}$ be constant in time. Then $\mathbf{z}\left(u^{i}\right)$ is concave in $u^{i}$. (ii) Let $u^{i}$ be a threshold policy with threshold parameter $\sigma$. Then $\mathbf{z}\left(u^{i}\right)$ is concave in $\sigma$.

Proof. We recall that a convex increasing function of a convex function is convex, and that a concave increasing function of a concave function is concave. We prove (ii). (i) is obtained in the same way. Let $u^{i}$ be a threshold policy with parameter $s$. We have

and thus

$$
Z_{t}:=\int_{0}^{t} u_{r}^{i} d r=\min (s, t)
$$

$$
V(t):=\log \left(\gamma\left(Z_{t}\right)\right)=\max [\log (\gamma(t)), \log (\gamma(s))]
$$

Since $\log \left(\gamma\left(Z_{t}\right)\right)$ is concave decreasing in $Z_{t}$, it is concave decreasing in $s$. Now

$$
w(s):=\int_{0}^{\tau} V(t) d t=\int_{0}^{\tau} \max [\log (\gamma(t)), \log (\gamma(s))] d t
$$

is concave decreasing in $s$ since it is the integral (over $t$ ) of concave decreasing functions in $s$. Thus $n w(s)$ is concave decreasing in $s$. Since $1-\exp$ is concave decreasing, then $1-\exp (n w(s))$ is concave increasing. Since log is concave increasing, we obtain the concavity of $\mathbf{z}$.

Theorem 2. Assume that the system is initially empty. Then

(i) the policy that achieves the maximum in $P 1$ is one whose components $u^{i}$ are all equal.

(ii) Each component $u^{i}$ of the policy $u$ that achieves the maximum in P2 is a threshold policy, and the thresholds $s$ are the same for all $i$.

Proof. Let $u^{i}$ be given static policies. We have by Jensen's inequality

$$
\frac{\log F_{D}(u)}{K}=\frac{\sum_{j=1}^{K} \log \mathbf{z}\left(\tau u^{j}\right)}{K} \leq \log \mathbf{z}\left(\frac{\sum_{j=1}^{K} \tau u^{j}}{K}\right)
$$

with equality holding above only when $u^{i}$ is the same for all $i$. This establishes (i).

(ii) The fact that one may restrict to threshold policies for each $i$ follows by applying the proof of Theorem 1 to each $u^{i}$. So now let $s(i)$ be the threshold used by policy $u^{i}$. Then we have again by Jensen's inequality

$$
\frac{\log F_{D}(u)}{K}=\frac{\sum_{j=1}^{K} \log \mathbf{z}(s(j))}{K} \leq \log \mathbf{z}\left(\frac{\sum_{j=1}^{K} s(j)}{K}\right) .
$$

with equality holding above only when $s(j)$ is the same for all $j$. 


\subsection{Adding Redundant Chunks}

We add $H$ redundant chunks and code them in a way to ensure that the reception of any $K$ chunks out of the $K+H$ ones is sufficient for the destination to be able to reconstruct the whole message.

Let $S_{n, p}$ be a binomially distributed r.v. with parameters $n$ and $p$, i.e.

$$
P\left(S_{n, p}=m\right)=B(p, n, m):=\left(\begin{array}{c}
n \\
m
\end{array}\right) p^{m}(1-p)^{n-m}
$$

The probability of a successful delivery of the message by time $\tau$ is thus

$$
P_{s}(\tau, K, H)=\sum_{j=K}^{K+H} B\left(\mathbf{z}\left(Z_{j}\right), K+H, j\right), \quad \text { where } \quad Z_{i}:=\int_{0}^{t} u_{s}^{i} d s .
$$

Theorem 3. Assume that the system is initially empty and assume that information is coded in a way that from any $K$ different chunks out of $K+H$ different chunks, the destination can reconstruct the whole original message (that was composed by $K$ initial chunks), Then

(i) the policy that achieves the maximum in $P 1$ is one whose components $u^{i}$ are all equal.

(ii) each component $u^{i}$ of the policy $u$ that achieves the maximum in P2 is a threshold policy, and the thresholds $s$ are the same for all $i$.

Proof: Let $A(K, H)$ be the set of subsets $h \subset\{1, \ldots, K+H\}$ that contain at least $K$ elements. For example, $\{1,2, \ldots, K\} \in A(K, H)$. Fix $p_{i}$ such that $\sum_{i=1}^{K+H} p_{i}=u$. Then the probability of successful delivery by time $\tau$ is given by

$$
P_{s}(\tau, K, H)=\sum_{h \in A(H, K)} \prod_{i \in h} \mathbf{z}\left(Z_{i}\right)
$$

For any $i$ and $j$ in $\{1, \ldots, K+H\}$ we can write

$$
P_{s}(\tau, K, H)=\mathbf{z}\left(Z_{i}\right) \mathbf{z}\left(Z_{j}\right) \phi_{1}+\left(\mathbf{z}\left(Z_{i}\right)+\mathbf{z}\left(Z_{j}\right)\right) \phi_{2}+\phi_{3}
$$

where $\phi_{1}, \phi_{2}$ and $\phi_{3}$ are nonnegative functions of $\left\{Z\left(p_{m}\right), m \neq i, m \neq j\right\}$. For example,

$$
\phi_{1}=\sum_{h \in A_{\{i, j\}}(H, K)} \prod_{\substack{m \in h \\ m \neq i, m \neq j}} \mathbf{z}\left(Z_{m}\right)
$$

where $A_{v}(K, H)$ is the set of subsets $h \subset\{1, \ldots, K+H\}$ that contain at least $K$ elements and such that $v \subset h$.

Now consider maximizing $F_{D}(\tau, K, H)$ over $Z_{i}$ and $Z_{j}$.

Assume that $Z_{i}^{\prime} \neq Z_{j}^{\prime}$. Since $\mathbf{z}(\cdot)$ is strictly concave, it follows by Jensen's inequality that $\mathbf{z}\left(Z_{i}^{\prime}\right)+\mathbf{z}\left(Z_{j}^{\prime}\right)$ can be strictly improved by replacing $Z_{i}^{\prime}$ and $Z_{j}^{\prime}$ by $Z_{i}=Z_{j}=\left(Z_{i}^{\prime}+Z_{j}^{\prime}\right) / 2$. This is also the unique maximum of the product $\mathbf{z}\left(Z_{i}\right) \mathbf{z}\left(Z_{j}\right)$ (using again Jensen's inequality applied to $\log \mathbf{z}$ ) and hence of 
$F_{D}(\tau, K, H)$. This holds for any $i$ and $j$ and for any $p^{\prime} \leq p$, hence it implies the Theorem.

The optimality of a symmetric choice of the transmission probabilities of chunks has a direct implication on the monotonicity of the performance with respect to the number $H$ of redundant chunks.

Fix any $\tau, K$ and $H$. Let $p^{*}$ be the optimal probability of sending a chunk of type $i$ for these given $\tau, K$ and $H$. (As we saw, this does not depend on $i$ ). The success probability under $p^{*}$ is the same as when increasing the redundancy to $H+1$ and using the vector $\left(p^{*}, \ldots, p^{*}, 0\right)$. Thus the utility when using $p^{*}$ for a given $\tau, K$ and $H$ is the same as when we increase the redundancy to $H+1$ and use the vector $\left(p^{*}, \ldots, p^{*}, 0\right)$. By definition, the latter is smaller than the optimal utility for the problem with $\tau, K, H+1$. We thus conclude that the utility is increasing in the amount of redundancy of chunks.

\section{Asymptotic approximations}

\subsection{The message model}

We study the model of dynamic transmission control (by the source) of Section 2; we shall consider the limit of the model (appropriately scaled) as the population size grows to infinity.

Denote $\bar{\lambda}=n \lambda$ ( $n$ is the population size). Then for any population size $n$, $w_{t}$ is the solution of the linear differential equation:

$$
\dot{w}_{t}=u_{t} \bar{\lambda}\left(1-w_{t}\right) \quad \text { or equivalently, } \quad \frac{d E\left[X_{t}\right]}{d t}=u_{t} \lambda\left(n-E\left[X_{t}\right]\right) .
$$

Bound. Dente $\xi_{t}(n):=X_{t}(n) / n$ where we add explicitly $n$ in the notation in order to stress the dependence of $X_{t}$ on $n$. By Jensen's inequality we have

$$
F_{D}(\tau, n)=1-E\left[\exp \left(-\bar{\lambda} \int_{0}^{\tau} \xi_{t}(n) d t\right)\right] \leq 1-\exp \left(-\bar{\lambda} \int_{0}^{\tau} w_{t} d t\right) .
$$

Tightness. We show that the bound is tight as $n$ grows. Consider a sequence of systems where the $n$th DTN has $n$ mobiles and where the contact process in the $n$th system is given by $\lambda(n)=\bar{\lambda} / n$.

Note that $w_{t}=E\left[\xi_{t}(n)\right]$ does not depend on $n$. We shall establish that

$$
\lim _{n \rightarrow \infty} F_{D}(\tau, n)=1-\lim _{n \rightarrow \infty} E\left[\exp \left(-\bar{\lambda} \int_{0}^{\tau} \xi_{t}(n) d t\right)\right]=1-\exp \left(-\bar{\lambda} \int_{0}^{\tau} w_{t} d t\right) .
$$

By the Strong Law of Large Numbers we have P-a.s.

$$
\lim _{n \rightarrow \infty} \xi_{t}(n)=\lim _{n \rightarrow \infty} \frac{\sum_{i=1}^{n} \zeta_{t}(i)}{n}=w_{t}
$$


It then follows from the bounded convergence theorem that $\lim _{n \rightarrow \infty} \int_{0}^{\tau} \xi_{t}(n) d t=$ $\int_{0}^{\tau} w_{t} d t$. Using again the bounded convergence Theorem we see that the corresponding LST converge to the LST of the limit, i.e.

$$
\lim _{n \rightarrow \infty} E\left[\exp \left(-\bar{\lambda} \int_{0}^{\tau} \xi_{t}(n) d t\right)\right]=\exp \left(-\bar{\lambda} \int_{0}^{\tau} w_{t} d t\right)
$$

which establishes the tightness of the bound.

\subsection{The chunk model}

We extend the above model to the case of transmission of chunks which we introduced in Section 7. If we denote by $\zeta_{t}^{j}(i)$ the indicator that the $j$ th mobile among the $n$ receives chunk $j$ during $[0, \mathrm{t}]$, then $X_{t}^{j}=\sum_{i=1}^{n} \zeta_{t}^{j}(i)$. We next define

$$
\xi_{t}^{j}(n):=\frac{X_{t}^{j}(n)}{n}, \quad w_{t}^{j}=E\left[\zeta_{t}^{j}(i)\right]=1-\exp \left(-\lambda \int_{0}^{\tau} u_{s}^{j} d s\right)
$$

As a direct extension of the case of message transfers, we have the following bound due to Jensen's inequality:

$$
F_{D}=\prod_{j=1}^{K}\left(1-E\left[\exp \left(-\lambda \int_{0}^{\tau} X_{t}^{j} d t\right)\right]\right) \leq \prod_{j=1}^{K}\left(1-\left[\exp \left(-\lambda \int_{0}^{\tau} w_{t}^{j} d t\right)\right]\right)
$$

Here again we can show as for the message-based model that the above bound becomes tight as the number of players $n$ increases.

\section{Concluding Comments}

This paper is part of our intensive ongoing research on performance issues and optimal control of Delay Tolerant Networks (DTNs). Most of the existing analysis of DTNs uses the mean field asymptotics to obtain explicit expressions and to solve optimal control issues, or it uses Markov chain techniques which often do not allow to obtain exact close form formulas for the steady state probabilities. In obtaining these close form expressions we are able to carry the investigation of DTNs on and solve various optimal control problems as well as non-cooperative games.

\section{Acknoledgements}

This work has been partially supported by the European Commission within the framework of the BIONETS project, IST-FET-SAC-FP6-027748, www.bionets.eu. It was also supported in part by the Arc Popeye collaborative project of INRIA. 


\section{References}

1. A. Al-Hanbali, P. Nain, and E. Altman, "Performance of Ad Hoc Networks with Two-Hop Relay Routing and Limited Packet Lifetime", First International Conference on Performance Evaluation Methodologies and Tools (Valuetools), Pisa, 2006.

2. Eitan Altman, Tamer Basar and Francesco De Pellegrini, "Optimal monotone forwarding policies in delay tolerant mobile Ad-Hoc networks", Inter-Perf 2008: Workshop on Interdisciplinary Systems Approach in Performance Evaluation and Design of Computer \& Communication Systems, Athens, Greece, October, 2008.

3. Eitan Altman, Francesco De Pellegrini. "Forward Correction and Fountain codes in Delay Tolerant Networks", IEEE Infocom, Rio de Janeiro, Brazil, April 19-25, 2009.

4. Eitan Altman, Giovanni Neglia, Francesco De Pellegrini, Daniele Miorandi. "Decentralized Stochastic Control of Delay Tolerant Networks", IEEE Infocom, Rio de Janeiro, Brazil, April 19-25, 2009

5. Vijay Erramilli, Augustin Chaintreau, Mark Crovella and Christophe Diot, "Diversity of forwarding paths in pocket switched networks", Proceedings of the 7th ACM SIGCOMM conference on Internet measurement, San Diego, California, USA, pp $161-174,2007$

6. A. W. Marshall and I. Olkin, Inequalities: Theory of Majorization and its Applications. Academic Press, 1979, vol. 143 of Mathematics in Science and Engineering

7. Peiaposen Luo, Hongyu Huang, Wei Shu, Minglu Li, Min-You Wu, "Performance Evaluation of Vehicular DTN Routing under Realistic Mobility Models", Wireless Communications and Networking Conference, WCNC 2008. March 31 - April 3, pp 2206-2211, 2008.

\section{Appendix: Majorization and Schur concavity}

Definition 1. (Majorization and Schur-Concave Function [6])

Consider two $n$-dimensional vectors $d(1), d(2)$. $d(2)$ majorizes $d(1)$, which we denote by $d(1) \prec d(2)$, if $\sum_{i=1}^{n} d_{[i]}(1)=\sum_{i=1}^{n} d_{[i]}(2)$ and

$$
\sum_{i=1}^{k} d_{[i]}(1) \leq \sum_{i=1}^{k} d_{[i]}(2), \quad k=1, \ldots, n-1, \quad \text { and }
$$

where $d_{[i]}(m)$ is a permutation of $d_{i}(m)$ satisfying $d_{[1]}(m) \geq d_{[2]}(m) \geq \ldots \geq$ $d_{[n]}(m), m=1,2$.

A function $f: R^{n} \rightarrow R$ is Schur concave if $d(1) \prec d(2)$ implies $f(d(1)) \geq$ $f(d(2))$. It is strictly Schur concave if strict inequality holds whenever $d(1)$ is not a permutation of $d(2)$.

Lemma 5. [6, Chapter 3] Assume that a function $g: R^{n} \rightarrow R$ can be written as the sum $g(d)=\sum_{i=1}^{n} \psi\left(d_{i}\right)$ where $\psi$ is a concave (resp. strictly concave) function from $R$ to $R$. Then $g$ is Schur (resp. strictly) concave. 